\title{
High-accuracy determination of the neutron flux in the new experimental area $n_{-}$TOF-EAR2 at CERN
}

M. Sabaté-Gilarte ${ }^{1,2}$, M. Barbagallo ${ }^{3, a}$, N. Colonna ${ }^{3}$, F. Gunsing ${ }^{4}$, P. Žugec $^{5}$, V. Vlachoudis ${ }^{1}$, Y.H. Chen ${ }^{6}$, A. Stamatopoulos ${ }^{7}$, J. Lerendegui-Marco ${ }^{2}$, M.A. Cortés-Giraldo ${ }^{2}$, A. Villacorta ${ }^{8}$, C. Guerrero ${ }^{2}$, L. Damone ${ }^{3}$, L. Audouin ${ }^{6}$, E. Berthoumieux ${ }^{4}$, L. Cosentino ${ }^{9}$, M. Diakaki ${ }^{4}$, P. Finocchiaro ${ }^{9}$, A. Musumarra ${ }^{9,10}$, T. Papaevangelou $^{4}$, M. Piscopo ${ }^{9}$, L. Tassan-Got ${ }^{6}$, O. Aberle ${ }^{1}$, J. Andrzejewski ${ }^{11}$, V. Bécares ${ }^{12}$, M. Bacak ${ }^{1,13}$, R. Baccomi ${ }^{14}$, J. Balibrea ${ }^{12}$, S. Barros ${ }^{15}$, F. Bečváŕr ${ }^{16}$, C. Beinrucker ${ }^{17}$, F. Belloni ${ }^{3}$, J. Billowes ${ }^{18}$, D. Bosnar ${ }^{5}$, M. Brugger ${ }^{1}$, M. Caamaño $^{19}$, F. Calviño ${ }^{20}$, M. Calviani ${ }^{1}$, D. Cano-Ott ${ }^{12}$, R. Cardella $^{1}$, A. Casanovas ${ }^{20}$, D.M. Castelluccio ${ }^{21,22}$, F. Cerutti $^{1}$, E. Chiaveri ${ }^{1}$, G. Cortés $^{21}$, K. Deo ${ }^{19}$, C. Domingo-Pardo ${ }^{23}$, R. Dressler ${ }^{24}$, E. Dupont ${ }^{4}$, I. Durán ${ }^{19}$, B. FernándezDomínguez $^{19}$, A. Ferrari ${ }^{1}$, P. Ferreira ${ }^{15}$, R.J.W. Frost ${ }^{18}$, V. Furman ${ }^{25}$, K. Göbel ${ }^{17}$, A.R. García ${ }^{12}$, A. Gawlik ${ }^{11}$, I. Gheorghe ${ }^{26}$, T. Glodariu ${ }^{26}$, I.F. Gonçalves ${ }^{15}$, E. González ${ }^{12}$, A. Goverdovski ${ }^{27}$, E. Griesmayer ${ }^{13}$, H. Harada ${ }^{28}$,

T. Heftrich ${ }^{17}$, S. Heinitz ${ }^{24}$, A. Hernández-Prieto ${ }^{1,20}$, J. Heyse ${ }^{29}$, D.G. Jenkins ${ }^{30}$, E. Jericha ${ }^{13}$, F. Käppeler ${ }^{31}$,

Y. Kadi ${ }^{1}$, T. Katabuchi ${ }^{32}$, P. Kavrigin ${ }^{13}$, V. Ketlerov ${ }^{27}$, V. Khryachkov ${ }^{27}$, A. Kimura ${ }^{28}$, N. Kivel ${ }^{24}$, M. Kokkoris ${ }^{7}$, M. Krtička ${ }^{16}$, E. Leal-Cidoncha ${ }^{19}$, C. Lederer ${ }^{33}$, H. Leeb ${ }^{13}$, M. Licata ${ }^{22,34}$, S. Lo Meo ${ }^{21,22}$, S.J. Lonsdale ${ }^{33}$, R. Losito ${ }^{1}$, D. Macina ${ }^{1}$, J. Marganiec ${ }^{11}$, T. Martínez ${ }^{12}$, C. Massimi ${ }^{22,34}$, P. Mastinu ${ }^{35}$, M. Mastromarco ${ }^{3}$, F. Matteucci ${ }^{14,36}$, E.A. Maugeri ${ }^{24}$, E. Mendoza ${ }^{12}$, A. Mengoni ${ }^{21}$, P.M. Milazzo ${ }^{14}$, F. Mingrone ${ }^{22,1}$, M. Mirea ${ }^{26}$, S. Montesano ${ }^{1}$, R. Nolte $^{37}$, A. Oprea ${ }^{26}$, F.R. Palomo-Pinto ${ }^{38}$, C. Paradela ${ }^{19}$, N. Patronis ${ }^{39}$, A. Pavlik ${ }^{40}$, J. Perkowski ${ }^{11}$, J.I. Porras ${ }^{41}$, J. Praena ${ }^{2,41}$, J.M. Quesada ${ }^{2}$, K. Rajeev ${ }^{42}$, T. Rauscher ${ }^{43,44}$, R. Reifarth ${ }^{17}$, A. Riego-Perez ${ }^{20}$, M.S. Robles ${ }^{19}$, P.C. Rout ${ }^{42}$, C. Rubbia ${ }^{1}$, J.A. Ryan ${ }^{18}$, A. Saxena ${ }^{42}$, P. Schillebeeckx ${ }^{29}$, S. Schmidt ${ }^{17}$, D. Schumann ${ }^{24}$, P. Sedyshev ${ }^{25}$, A.G. Smith ${ }^{18}$, S.V. Suryanarayana ${ }^{42}$, G. Tagliente ${ }^{3}$, J.L. Tain ${ }^{23}$, A. Tarifeño-Saldivia ${ }^{20,23}$, A. Tsinganis ${ }^{7}$, S. Valenta $^{16}$, G. Vannini ${ }^{2,34}$, V. Variale ${ }^{3}$, P. Vaz ${ }^{15}$, A. Ventura ${ }^{22}$, R. Vlastou ${ }^{7}$, A. Wallner ${ }^{45}$, S. Warren ${ }^{18}$, M. Weigand ${ }^{17}$,

C. Wolf ${ }^{17}$, P.J. Woods ${ }^{33}$, C. Weiss ${ }^{1,13}$, and T. Wright ${ }^{18}$

1 European Organization for Nuclear Research (CERN), Geneva, Switzerland

2 Departamento de Física Atómica, Molecular y Nuclear, Universidad de Sevilla, Sevilla, Spain

3 Istituto Nazionale di Fisica Nucleare, Sezione di Bari, V. Orabona 4, 70125 Bari, Italy

${ }^{4}$ CEA Irfu, Université Paris-Saclay, F-91191 Gif-sur-Yvette, France

5 Department of Physics, Faculty of Science, University of Zagreb, Zagreb, Croatia

${ }^{6}$ Centre National de la Recherche Scientifique/IN2P3 - IPN, Orsay, France

7 National Technical University of Athens (NTUA), Athens, Greece

8 University of Salamanca, Salamanca, Spain

9 INFN Laboratori Nazionali del Sud, Catania, Italy

10 Dipartimento di Fisica, Università di Catania, Catania, Italy

11 University of Lodz, Lodz, Poland

12 Centro de Investigaciones Energéticas Medioambientales y Tecnológicas (CIEMAT), Madrid, Spain

13 Technische Universität Wien, Wien, Austria

14 Istituto Nazionale di Fisica Nucleare, Sezione di Trieste, Trieste, Italy

15 Instituto Superior Técnico, Lisbon, Portugal

16 Charles University, Prague, Czech Republic

17 Goethe University Frankfurt, Frankfurt, Germany

18 University of Manchester, Manchester, UK

19 University of Santiago de Compostela, Santiago de Compostela, Spain

20 Universitat Politècnica de Catalunya, Barcelona, Spain

21 Agenzia nazionale per le nuove tecnologie (ENEA), Bologna, Italy

22 Istituto Nazionale di Fisica Nucleare, Sezione di Bologna, Bologna, Italy

23 Instituto de Física Corpuscular, Universidad de Valencia, Valencia, Spain

24 Paul Scherrer Institut (PSI), Villingen, Switzerland

25 Joint Institute for Nuclear Research (JINR), Dubna, Russia

${ }^{26}$ Horia Hulubei National Institute of Physics and Nuclear Engineering, Măgurele, Romania

27 Institute of Physics and Power Engineering (IPPE), Obninsk, Russia

28 Japan Atomic Energy Agency (JAEA), Tokai-mura, Japan 
${ }^{29}$ European Commission, Joint Research Centre, Geel, Retieseweg 111, B-2440 Geel, Belgium

${ }^{30}$ University of York, York, UK

31 Karlsruhe Institute of Technology, Campus North, IKP, 76021 Karlsruhe, Germany

${ }^{32}$ Tokyo Institute of Technology, Tokyo, Japan

33 School of Physics and Astronomy, University of Edinburgh, Edinburgh, UK

34 Dipartimento di Fisica e Astronomia, Università di Bologna, Bologna, Italy

35 Istituto Nazionale di Fisica Nucleare, Sezione di Legnaro, Legnaro, Italy

36 Dipartimento di Astronomia, Università di Trieste, Trieste, Italy

37 Physikalisch-Technische Bundesanstalt (PTB), Bundesallee 100, 38116 Braunschweig, Germany

38 Depto. Ingeniería Electrónica, Escuela Técnica Superior de Ingenieros, Universidad de Sevilla, Sevilla, Spain

39 University of Ioannina, Ioannina, Greece

40 University of Vienna, Faculty of Physics, Vienna, Austria

41 University of Granada, Granada, Spain

42 Bhabha Atomic Research Centre (BARC), Mumbai, India

43 Centre for Astrophysics Research, University of Hertfordshire, Hatfield, UK

44 Department of Physics, University of Basel, Basel, Switzerland

45 Australian National University, Canberra, Australia

Received: 30 June 2017 / Revised: 31 August 2017

(c) Società Italiana di Fisica / Springer-Verlag 2017

Communicated by T. Motobayashi

\begin{abstract}
A new high flux experimental area has recently become operational at the $\mathrm{n}_{-} \mathrm{TOF}$ facility at CERN. This new measuring station, n_TOF-EAR2, is placed at the end of a vertical beam line at a distance of approximately $20 \mathrm{~m}$ from the spallation target. The characterization of the neutron beam, in terms of flux, spatial profile and resolution function, is of crucial importance for the feasibility study and data analysis of all measurements to be performed in the new area. In this paper, the measurement of the neutron flux, performed with different solid-state and gaseous detection systems, and using three neutronconverting reactions considered standard in different energy regions is reported. The results of the various measurements have been combined, yielding an evaluated neutron energy distribution in a wide energy range, from $2 \mathrm{meV}$ to $100 \mathrm{MeV}$, with an accuracy ranging from $2 \%$, at low energy, to $6 \%$ in the high-energy region. In addition, an absolute normalization of the $\mathrm{n}_{-}$TOF-EAR2 neutron flux has been obtained by means of an activation measurement performed with ${ }^{197} \mathrm{Au}$ foils in the beam.
\end{abstract}

\section{Introduction}

The neutron time-of-flight facility $\mathrm{n}_{-} \mathrm{TOF}^{1}$, operational since 2001 at the European Organization for Nuclear Research (CERN), is characterised by a high-intensity, highresolution, wide spectrum neutron beam mostly dedicated to measurements of neutron-induced cross sections of interest in nuclear technology [1,2], astrophysics [3,4] and more recently for medical applications $[5,6]$. The neutron beam is produced by spallation of a pulsed proton beam from the CERN Proton Synchrotron accelerator (PS), with momentum of $20 \mathrm{GeV} / \mathrm{c}$, impinging on a cylindrical lead target surrounded by water for cooling and neutron moderation purposes.

The neutron beam characteristics and state-of-the-art detectors and acquisition systems make n_TOF ideal for measuring radioisotopes, in particular, actinides, as well as for identifying and studying resonances in neutron cross sections. For the first 13 years of operation, only one experimental area was available, located at $185 \mathrm{~m}$ from the spallation target along the horizontal direction. In this area, now denoted as n_TOF-EAR1 (hereafter EAR1, Experimental Area 1), the neutron beam is characterised by a high instantaneous flux of $10^{6}$ neutrons/bunch, covering the energy range from $25 \mathrm{meV}$ to over $1 \mathrm{GeV}$, and a neutron energy resolution in a large part of the energy range of $\Delta E / E$ from $10^{-3}$ to $10^{-4}$. A more detailed description of the neutron beam features in EAR1 can be found in ref. [7].

In 2014, a new experimental hall located on the vertical direction at $20 \mathrm{~m}$ distance from the spallation target, the so-called n_TOF-EAR2 [8,9] (hereafter EAR2, Experimental Area 2), was completed and became operational. The main advantage of this new measuring station with respect to the existing one consists in a flux on average 40 times higher than in EAR1, a convenient feature that makes it possible to perform challenging new measurements. Combined with the shorter time-of-flight at a given energy, 10 times lower than EAR1 due to the shorter flight-path, the higher flux results in more than two orders of magnitude higher signal-to-background ratio, when considering the background related to the natural radio-

\footnotetext{
a e-mail: massimo.barbagallo@ba.infn.it

1 www.cern.ch/ntof.
} 
activity of unstable isotopes, which represents the dominant component for short-lived radionuclides. These features of the EAR2 neutron beam open the way to measurements of neutron-induced reactions on very thin samples, radioactive isotopes with short half-life or reactions of low cross sections [10].

Following the completion of the second experimental area, a large effort was devoted to the commissioning of the new neutron beam, with a series of dedicated measurements performed with the aim of characterizing it in terms of flux and its energy dependence, spatial beam profile, resolution function and background. An accurate knowledge of all these quantities is in fact fundamental for the envisaged vast experimental program in EAR2 on neutron-induced reaction cross sections for fundamental and applied nuclear physics.

In this work we report on dedicated measurements of the neutron flux, carried out as part of the commissioning of the new experimental area. To determine the flux with high accuracy in a wide energy range, from $2 \mathrm{meV}$ to $100 \mathrm{MeV}$, a combination of several detection systems and neutron converting reactions, considered standards, were used. In this way, the systematic uncertainties related to the experimental technique were minimised. The campaign extended over two running periods, with some measurements repeated with different samples for a higher accuracy.

This paper is organized as follows: in sect. 2 the experimental setups are described, while in sect. 3 the procedure used in the data analysis is discussed. The final results are presented in sect. 4 .

\section{The experimental setup for neutron flux determination at $\mathbf{n}_{-}$TOF}

The time-of-flight technique (TOF) is used to determine the kinetic energy of neutrons $\left(E_{n}\right)$ from the spallation process by the flight time between the production point and the detection system. The neutron beam in a TOF facility is characterised by the neutron fluence $\left(F_{E}\right)$ and the neutron flux $\left(\Phi\left(E_{n}\right)\right)$ spectra that, according to the ICRU recommendations [11], are defined as

$$
F_{E}=\frac{\mathrm{d} N_{E}}{\mathrm{~d} t \cdot \mathrm{d} a} \quad \text { and } \quad \Phi\left(E_{n}\right)=\frac{\mathrm{d} N_{E}}{\mathrm{~d} t} .
$$

$\mathrm{d} N_{E}$ denotes the density distribution of neutrons reaching the sample with energy between $E_{n}$ and $E_{n}+\mathrm{d} E_{n}, \mathrm{~d} t$ the time element and $\mathrm{d} a$ the cross-sectional area of an elemental sphere.

Nevertheless, when talking about TOF facilities the term flux is used to designate what in the ICRU report is termed fluence rate (time-differential). Therefore, $\Phi\left(E_{n}\right)$ will be hereafter defined as the number of neutrons reaching the sample by element of energy, area and time. At $\mathrm{n}_{-}$TOF, where the spatial profile of the neutron beam is not uniform and neutrons are delivered in bunches at very low repetition rate $(<0.8 \mathrm{~Hz})$, it is more convenient to
Table 1. Neutron induced reactions used in this work to characterise the neutron flux in EAR2 at n_TOF, and the energy range in which the respective cross sections are considered standard. The last reaction is important to obtain the absolute value of the flux by means of an activation measurement.

\begin{tabular}{cc}
\hline Reaction & Standard energy range \\
\hline${ }^{6} \mathrm{Li}(n, t)$ & $0.0253 \mathrm{eV}$ to $1 \mathrm{MeV}$ \\
${ }^{10} \mathrm{~B}(n, \alpha)$ & $0.0253 \mathrm{eV}$ to $1 \mathrm{MeV}$ \\
${ }^{235} \mathrm{U}(n, f)$ & $0.0253 \mathrm{eV}$ and $0.15-200 \mathrm{MeV}$ \\
${ }^{197} \mathrm{Au}(n, \gamma)$ & $0.0253 \mathrm{eV}$ and $0.2-2.5 \mathrm{MeV}$ \\
\hline
\end{tabular}

consider the total number of neutrons in a bunch integrated over the whole beam spatial profile [12]. This magnitude can be only obtained experimentally from $F_{E}$ as a discrete function by summing-up all the neutron-induced reactions that have been detected for a reference sample in sequential finite time-slots (or histogram bins) inside the neutron spills. The knowledge of this quantity, i.e. the total number of neutrons impinging on the sample in the whole measurement, is fundamental for the determination of the reaction cross section.

The neutron flux is measured by means of neutron induced reactions whose cross sections are smooth, large and accurately known in specific energy regions, and for this reason generally adopted as standard $[13,14]$.

In table 1 the three reactions used in this work are listed together with the cross section used as a reference for the activation measurement. The wide energy range of the n_TOF neutron beam makes it mandatory to use different standards, covering the full energy range. Furthermore, the combination of various detection systems based on different working principles allows minimising possible sources of uncertainties such as, for example, the detection efficiency.

The following detectors have been used for the characterization of the EAR2 neutron flux in two campaigns.

1) A low-mass Silicon Monitor, SiMon2 [15], similar to the one used in the first experimental area and described in ref. [16]. Apart from the flux measurement, it is permanently installed in the neutron beam to continuously monitor the flux and to provide the fluence in any measurement. It consists of a set of four silicon pad detectors located outside the beam, surrounding a foil with a deposit of ${ }^{6} \mathrm{LiF}$. The system is operated inside a vacuum chamber. The whole setup is placed at the entrance of EAR2, at a short distance from the last collimator. The silicon detectors, from Micron Semiconductors (MSX09-300), have a surface of $3 \times 3 \mathrm{~cm}^{2}$ and a thickness of $300 \mu \mathrm{m}$. These dimensions were selected as a compromise between a high efficiency and low capacitance, to ensure a low-noise operation with standard preamplifiers. The energy resolution of the detectors results in a perfect separation between tritons and $\alpha$-particles emitted in the ${ }^{6} \mathrm{Li}(n, t) \alpha$ reaction, and a good rejection of the electronic noise and of the gamma background. Two different samples with ${ }^{6} \mathrm{LiF}$ deposit were used for the flux measurement in EAR2: the first one, with an areal density of $420 \mu \mathrm{g} / \mathrm{cm}^{2}$, was used 
only for the high-energy region, as it was affected by pileup problems at energies below $1 \mathrm{eV}$, where both the flux and the cross section are high. A thinner $105 \mu \mathrm{g} / \mathrm{cm}^{2}$ thick deposit was later used in order to extend the measurement down to the meV neutron energy region.

2) A gaseous detector, based on the MicroMegas microbulk technology [17-19]. This type of detectors is being used at n_TOF since many years for various purposes, due to their high signal-to-background ratio, high radiation resistance and low mass, which minimises perturbation of the neutron beam. A Micromegas is defined by two gas volumes: the drift volume, typically of a few millimeter thickness, and the amplification region, $50 \mu \mathrm{m}$, separated by a $5 \mu \mathrm{m}$ micro-mesh layer. The neutron-converting material is deposited on the cathode, in the drift region, delimited by the cathode and the mesh. In this region the ionization from the charged products of the neutron reactions takes place. The secondary electrons drift through the electric field, $\sim 1 \mathrm{kV} / \mathrm{cm}$, to the mesh. Due to the high electric field in the second gap, $\geq 10 \mathrm{kV} / \mathrm{cm}$, an avalanche multiplication of the primary electrons takes place.

Two different detector geometries were implemented. In the first one, hereafter referred to as $M G A S$, electrodes $9.5 \mathrm{~cm}$ in diameter were used equipped with deposits of $93 \mu \mathrm{g} / \mathrm{cm}^{2}$ enriched ${ }^{235} \mathrm{U}$ and with $20 \mathrm{~nm}$-thick deposit of ${ }^{10} \mathrm{~B}_{4} \mathrm{C}$, on 30 and $18 \mu \mathrm{m}$-thick aluminium backings respectively. The entrance and exit windows of the aluminium chamber which contains the setup were made of $25 \mu \mathrm{m}$ thick kapton foils. The second chamber, referred to as monMGAS, contains electrodes $6 \mathrm{~cm}$ in diameter and aluminized mylar windows; it was loaded with a $281 \mu \mathrm{g} / \mathrm{cm}^{2}$ thick deposit of ${ }^{235} \mathrm{U}$ with an enrichment of $99.9 \%$ on a $30 \mu \mathrm{m}$ thick aluminium foil. In both cases, the chamber was filled with a mixture of $88 \% \mathrm{Ar}, 10 \% \mathrm{CF}_{4}$ and $2 \%$ $\mathrm{iC}_{4} \mathrm{H}_{10}$ at atmospheric pressure.

3) Finally, a set of position-sensitive Parallel Plate Avalanche Counters (PPAC [20,21]) equipped with a $70 \mu \mathrm{g} / \mathrm{cm}^{2}$ thick ${ }^{235} \mathrm{U}$ deposit on an aluminium backing of $0.7 \mu \mathrm{m}$ thickness were employed. The detectors are mounted on both sides of the deposit to detect fission fragments in coincidence, a technique that results in a very low background from $\alpha$-particles from the natural radioactivity of the sample, and allows one to reconstruct the neutron interaction position. Each PPAC consists of a central anode, for timing, flanked by two position-sensitive cathodes. Three PPACs working at low gas pressure were developed in order to measure the flux and the beam profile. The detectors were hosted in a chamber specifically designed for operation in EAR2. The fission fragments are identified by a coincidence window of $20 \mathrm{~ns}$. More details on the detectors and on the analysis procedure can be found in refs. [22,23].

In fig. 1 the two adopted experimental setups are presented.

The full waveforms of detector signals were acquired by the standard n_TOF Data Acquisition System, based on SPDevices ADQ412DC-3G cards of $2 \mathrm{GS} / \mathrm{s}$ maximum sampling rate, 12 bits resolution and 175 MBytes on-board memory. The special features of these cards ensure the collection of data for a time-of-flight corresponding to neu-

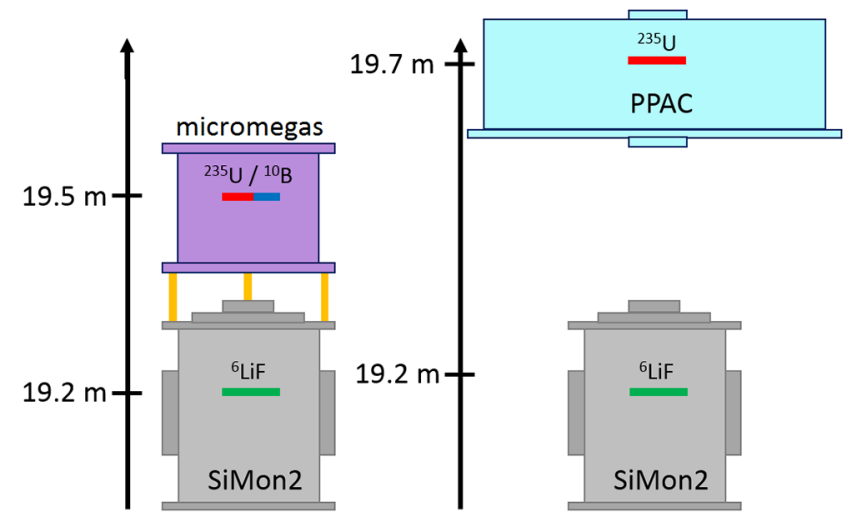

Fig. 1. Scheme of the experimental setup when micromegas (left) and PPAC (right) were used to measure the neutron flux. The SiMon2 chamber was always in place, located at $18.42 \mathrm{~m}$ from the spallation target. The flight paths of each neutron converter with respect to the spallation target are also indicated.

tron energies well below the thermal energy. The signals from SiMon2 and micromegas detectors are reconstructed by means of a Pulse Shape Analysis routine described in [24], while in the case of PPAC a dedicated routine was used. In all cases, information was extracted on the amplitude, area, timing and other pertinent quantities of the signals.

Together with these detection systems, two circular gold foils were also exposed to the beam in order to determine the absolute value of the neutron flux by activation. The two foils, placed back-to-back and covering fully the beam spot, were $100 \mu \mathrm{m}$ thick.

\section{Data analysis}

The reaction yield represents the probability for a neutron to undergo that reaction inside the sample. For thin targets, where the scattering in the deposit is negligible, the theoretical yield is defined by

$$
Y^{\mathrm{th}}\left(E_{n}\right)=\left(1-e^{-n \cdot \sigma_{t}\left(E_{n}\right)}\right) \cdot \frac{\sigma_{r}\left(E_{n}\right)}{\sigma_{t}\left(E_{n}\right)},
$$

where $n$ is the areal density (atoms/barn) of the target deposit, $\sigma_{r}$ and $\sigma_{t}$ are, respectively, the reaction and total cross sections for the isotope used as neutron converter. In this work, the evaluated cross sections from the ENDF/B-VII.1 library [25] were used for all the samples up to $20 \mathrm{MeV}$, while the IAEA reference file for the ${ }^{235} \mathrm{U}(n, f)$ cross section was adopted above this energy [14]. Experimentally, the yield is

$$
Y^{\exp }\left(E_{n}\right)=\frac{C\left(E_{n}\right)-B\left(E_{n}\right)}{\varepsilon\left(E_{n}\right) \cdot \Phi\left(E_{n}\right)} .
$$


The experimental value inside an energy bin is the integral of the detected events (counts), divided by the binwidth, and this value is assigned to the bin-center in the histogram. Taking this into account, in eq. (3), $C\left(E_{n}\right)$ represents the total recorded number of counts per bunch and $B\left(E_{n}\right)$ the background contribution in the bin centered at $E_{n}$, while $\varepsilon\left(E_{n}\right)$ is the efficiency for detecting the product of the neutron interaction. This factor includes the geometrical efficiency, the angular distribution and the kinematic effects for a given reaction. The uncertainty of the energy dependence of the neutron flux depends on all quantities that are a functions of the neutron energy, $E_{n}$ (such as the efficiency), while the absolute value of the flux is affected by energy-independent factors, such as the areal density of the deposit.

The relationship between the theoretical (2) and the experimental (3) reaction yield provides the neutron flux, $\Phi\left(E_{n}\right)$ :

$$
\Phi\left(E_{n}\right)=\frac{C\left(E_{n}\right)-B\left(E_{n}\right)}{\varepsilon\left(E_{n}\right) \cdot\left(1-e^{-n \cdot \sigma_{t}\left(E_{n}\right)}\right) \frac{\sigma_{r}\left(E_{n}\right)}{\sigma_{t}\left(E_{n}\right)}} .
$$

It is convenient to express the neutron flux as the total number of neutrons for a nominal proton pulse. Therefore, in eq. (4) the background-subtracted number of counts recorded in the whole measurement is divided by the total number of incident protons and multiplied by $7 \cdot 10^{12}$, a value that represents the nominal proton intensity for a dedicated pulse delivered by the PS to the n_TOF experiment.

The neutron kinetic energy in the equations above is determined from the time-of-flight according to the following expression:

$$
E_{n}(T)=m_{n} \cdot c^{2} \cdot\left(\frac{1}{\sqrt{1-\left(\frac{L+\lambda(T)}{c \cdot T}\right)^{2}}}-1\right),
$$

where $T$ is the calibrated time-of-flight reconstructed from the signal, $c$ the speed of light and $m_{n}$ the neutron mass. The time-of-flight is determined relative either from a pick-up signal or from the signal of the so-called $\gamma$-flash, generated in the detector by the prompt $\gamma$-rays and relativistic particles produced in the spallation process.

The quantity $\lambda(T)$ reflects the resolution function of the $\mathrm{n}_{-}$TOF neutron beam, i.e. the spread of the true neutron energy for a given time-of-flight, or equivalently the spread in time-of-flight for a given true neutron energy. Such a spread, caused by the stochastic moderation process neutrons undergo inside the spallation source and moderation circuit, can be expressed as an additional effective flight path that neutrons have to travel inside the lead target and the moderator system before entering the beam line. This quantity is not easily accessible experimentally, but can be determined as a function of the neutron energy by means of Monte Carlo simulations of the spallation process.

Studies of the resolution function for the second experimental area at $\mathrm{n}_{-} \mathrm{TOF}$ have been performed with FLUKA $[26,8]$ and GEANT4 $[27,28]$. They indicate that in the relatively wide energy range from a few $\mathrm{eV}$ to several tens of $\mathrm{keV}$, the average value of $\lambda$ remains approximately constant, so that a fixed value for the total effective flight path $L+\lambda$ can be used in eq. (5). On the contrary, below $1 \mathrm{eV}$ and above $100 \mathrm{keV}$ the resolution function shows large variations, both in terms of average value and width of the $\lambda$ distribution. If not properly included in the analysis, this behaviour would affect the reconstructed neutron energy and, as a consequence, the neutron flux. For the first experimental area the effect of the resolution function is relatively small at low and epithermal neutron energies, being $\Delta E / E$ of the order of $10^{-3}-10^{-4}$, thanks to the long flight path of EAR1 $(200 \mathrm{~m})$. For this reason, it was not considered in the neutron flux determination, while it is routinely included in resonance shape analysis, see for example [29]. On the contrary, in EAR2 the effect of the resolution function is much larger, reaching approximately $2 \%$ at thermal neutron energy, and has therefore to be taken into account in the flux determination. The only way to proceed in this respect is to convolute the theoretical yield of the three reference reactions, i.e. ${ }^{235} \mathrm{U}(n, f)$, ${ }^{6} \mathrm{Li}(n, t)$ and ${ }^{10} \mathrm{~B}(n, \alpha)$, with the simulated resolution function, before using it in eq. (4).

The method followed in this work is based on the resampling and propagation of neutrons scored on a tally surface just above the spallation target. More details on the propagation procedure can be found in $[8,28]$. Neutrons on the scoring plane are recorded with their true energy and time elapsed since the start of the spallation process (i.e., their time-of-flight inside the target-moderator assembly). When they are transported to the experimental area, only the time-of-flight is changed according to the geometrical distance between the scoring plane and the sample position in EAR2. For a given reference reaction, the yield is calculated on the basis of the true neutron energy and corresponding reference cross section, but assigned to an energy bin calculated on the basis of the time-of-flight. The resulting distribution, which takes into account the shift or spread in the reconstructed energy related to the resolution function, can at this point be used in eq. (4), instead of the point-wise cross sections, to extract the neutron flux. Before, however, an accurate value of the effective flight path to be used in the timeto-energy conversion of the experimental data has to be determined. This is done by comparing the simulated yield with the experimental one: the value of the flight path is adjusted and the simulated yield is recalculated in an iterative procedure until the position of the resonances in time-of-flight perfectly matches the experimental ones. In fig. 2, the expected yield of ${ }^{235} \mathrm{U}(n, f)$ based on the ENDF data library and the FLUKA-based resolution function is compared with the final experimental yield measured with monMGAS; the same comparison was performed for MGAS and PPAC. For the reactions that do not have any resonance in the range of interest, i.e. for the ${ }^{10} \mathrm{~B}(n, \alpha)$ and ${ }^{6} \mathrm{Li}(n, t)$ reactions, the effective flight path is calculated by simply considering, i.e. adding or subtracting, the geometrical distance between the position of the corresponding samples and the ${ }^{235} \mathrm{U}$ sample. 


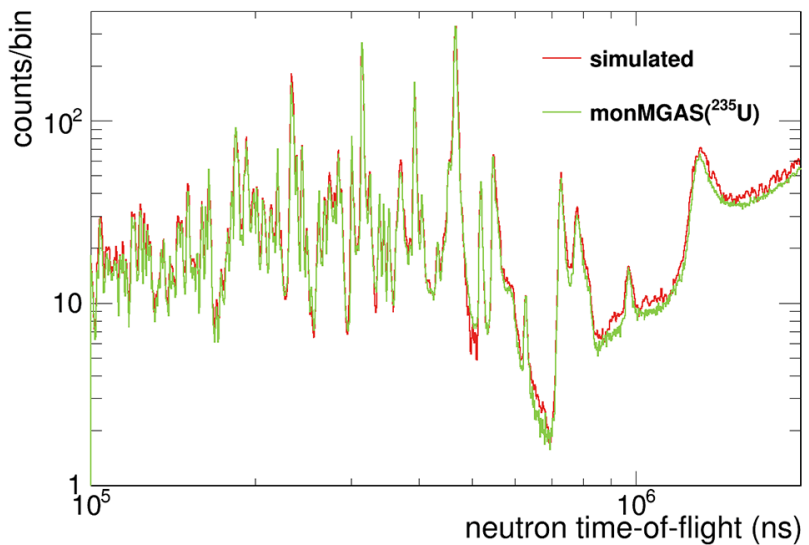

Fig. 2. Experimental (green) and calculated (red) ${ }^{235} \mathrm{U}(n, f)$ reaction yield in the resolved resonance region as a function of the neutron time-of-flight. The agreement between the two yields, obtained for a proper choice of the flight path length, demonstrates the accuracy of the time-to-energy calibration.

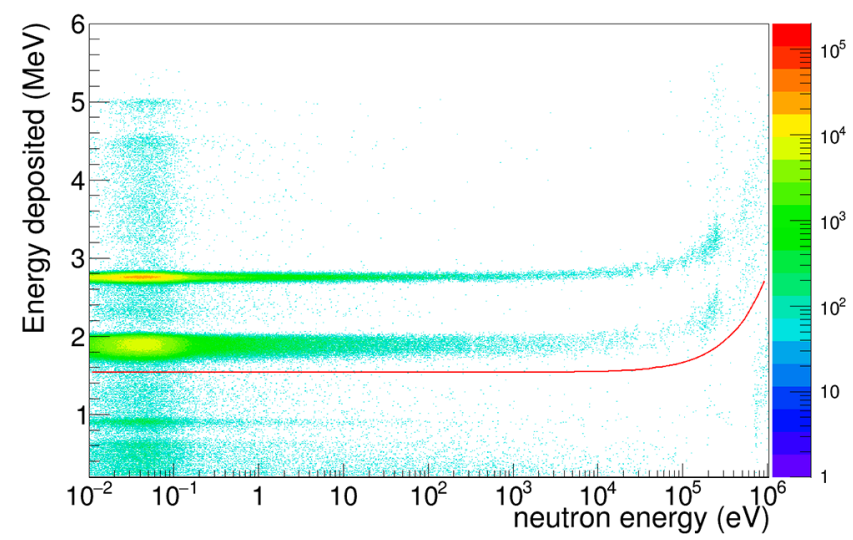

Fig. 3. Scatter plot of the energy deposited in a silicon detector of the SiMon2 apparatus, versus the reconstructed neutron energy. The red line corresponds to the cut applied to separate tritons and $\alpha$-particles from the background and the electronic noise of the detector.

\subsection{Analysis of SiMon2 with ${ }^{6} \mathrm{Li}$ converter}

The use of optimized electronics, in particular of specifically designed preamplifiers, in combination with the thin deposit results in a very good separation between tritons and $\alpha$-particles from the ${ }^{6} \operatorname{Li}(n, t) \alpha$ reaction, as well as between environmental background or electronic noise of the detector. Due to kinematic effects, the energy of the reaction products increases with the neutron energy. Therefore, the selection of tritons and $\alpha$-particles requires a non-linear cut on the $2 \mathrm{D}$ plot of the energy deposited in the silicon detector versus neutron energy. Figure 3 shows an example of the scatter 2D plot of the energy deposited by detected particles versus neutron energy for one of the silicon detectors, where the solid red line represents the 2-dimensional condition used in the analysis.

The efficiency of SiMon2 was estimated by means of GEANT4 simulations, on the basis of the geometrical de-

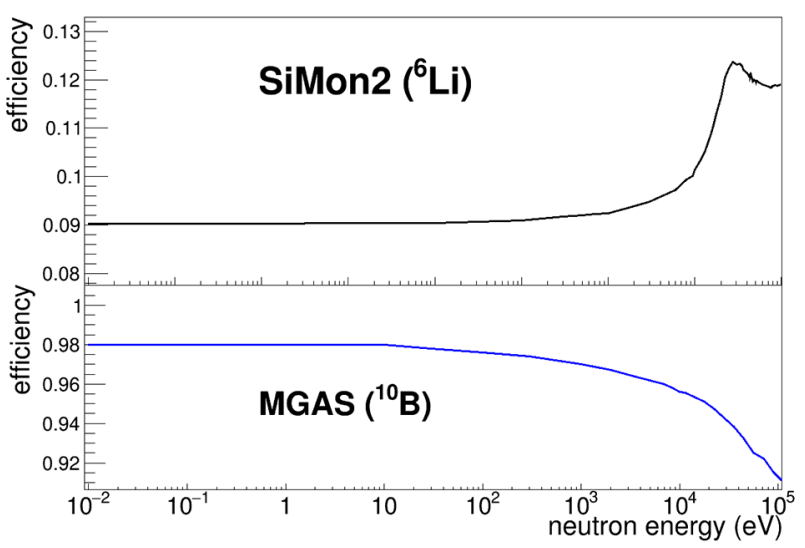

Fig. 4. (Top panel) SiMon2 efficiency for the detection of products from the ${ }^{6} \mathrm{Li}(n, t) \alpha$ reaction. (Bottom panel) Efficiency for detecting the products of the ${ }^{10} \mathrm{~B}(n, \alpha){ }^{7} \mathrm{Li}$ reaction in the MGAS detector.

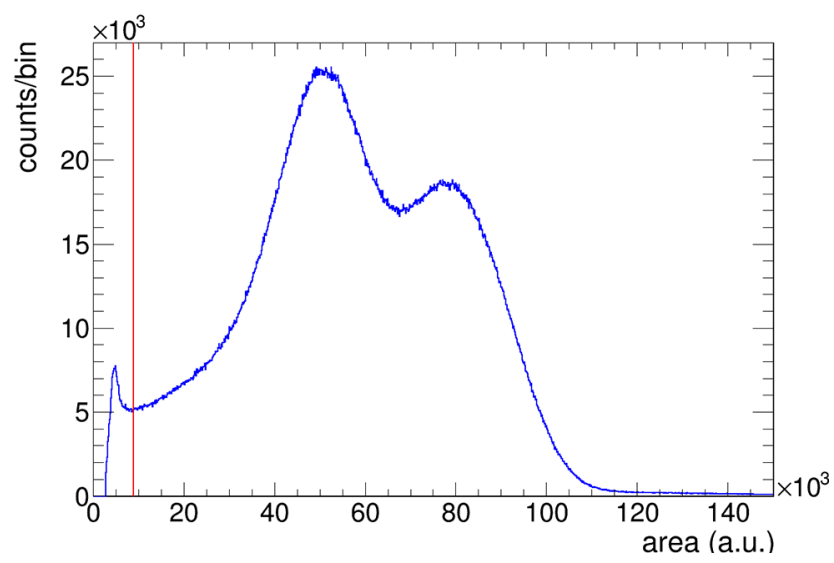

Fig. 5. Spectrum of the signal area for the ${ }^{235} \mathrm{U}(n, f)$ reaction measured with the monMGAS. The two bumps correspond to the light and heavy fission fragments. The $\alpha$-particles from the natural radioactivity of the sample are eliminated by the electronic threshold indicated by the red line.

tails described in ref. [15]. As shown in the top panel of fig. 4 , the efficiency is constant below $1 \mathrm{keV}(\sim 9 \%)$ while at higher energies the forward peaked angular distribution of tritons causes an increase of the efficiency. The intrinsic uncertainty in the angular distribution of the products of the ${ }^{6} \mathrm{Li}(n, t) \alpha$ reaction results in a relatively large uncertainty, of the order of $9 \%$, on the efficiency and hence of the extracted neutron flux above $10 \mathrm{keV}$.

\subsection{Flux determination with ${ }^{235} \mathrm{U}$ converter}

In order to determine the flux from the ${ }^{235} \mathrm{U}(n, f)$ reaction, three complementary detection systems were used: MGAS, monMGAS and PPAC. The high $Q$-value of the neutron induced fission reaction on ${ }^{235} \mathrm{U}$ results in a good discrimination of the fission fragments from the electronic noise and the background, due to the $\alpha$-activity of the uranium sample. For the monMGAS, the separation is shown in fig. 5, where the typical double-bump distribution is 


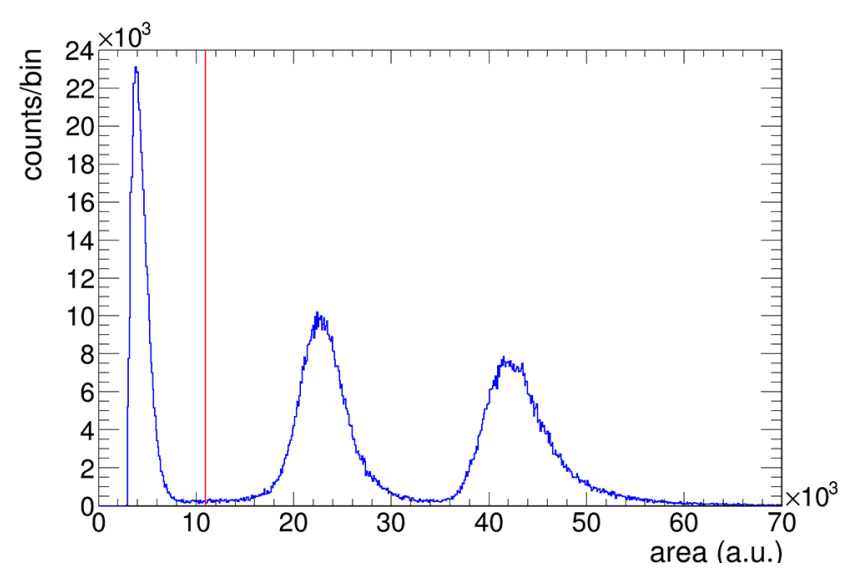

Fig. 6. Spectrum of the signal area from the ${ }^{10} \mathrm{~B}(n, \alpha)$ reaction measured with the MGAS detector. The peaks corresponding to the $\alpha$-particles and to ${ }^{7} \mathrm{Li}$ are well above the electronic noise, which is suppressed by the electronic threshold indicated by the red line. The energy resolution of the detector does not allow to separate the two final states of the reaction.

also visible. An energy-independent cut on the signal amplitude is sufficient to reject the background and select the fission fragments. The threshold is chosen to ensure maximum efficiency.

Due to the presence of a strong prompt signal, the socalled $\gamma$-flash, mostly induced by prompt $\gamma$-rays produced in the spallation target $[7,8]$, the maximum energy that can be reached with the micromegas detectors is of a few $\mathrm{MeV}$. On the contrary, PPACs are rather insensitive to $\gamma$-rays. Considering also that PPAC signals are very fast, the recovery time of the detector to the $\gamma$-flash is almost immediate (a few ns). These features bestow the system the possibility of extending the range of the measured flux up to $100 \mathrm{MeV}$ neutron energy.

According to Monte Carlo simulations of the setup, the efficiency for micromegas detectors is close to $95 \%$ in the energy range up to a few $\mathrm{MeV}$, while for the PPAC the efficiency is $\sim 60 \%$ up to a few $\mathrm{MeV}$, and changes above this value due to corrections for the angular anisotropy of the fragment emission [22].

As mentioned before, the calibration of the neutron energy, from the time-of-flight, is based on the analysis of the resonances in the ${ }^{235} \mathrm{U}(n, f)$ cross section.

\subsection{Analysis of MGAS with ${ }^{10} \mathrm{~B}$ target}

The ${ }^{10} \mathrm{~B}(n, \alpha){ }^{7} \mathrm{Li}$ reaction was measured with the MGAS. The energy resolution of the detector turned out to be not sufficient for separating the two exit channels of the reaction, i.e. the ground state $(6 \%)$ and the first excited stated (94\%), whose decay to the ground state is accompanied by the emission of a $478 \mathrm{keV} \gamma$-ray. Nevertheless, both reaction products are well separated from the electronic noise and the background, as indicated by the red line in fig. 6. As in the case of SiMon2, an energy-dependent cut has been applied on the deposited energy to select the $\alpha$-particles and the ${ }^{7} \mathrm{Li}$ ions above $1 \mathrm{keV}$.
The efficiency of the detector was estimated by means of Monte Carlo simulations of the energy loss in the sample and in the gas volume. The angular distribution of both products, reported in ref. [30], as well as the effect of the bleed-through of ${ }^{7} \mathrm{Li}$ below the threshold were taken into account in the efficiency calculation. As shown in the bottom panel of fig. 4, the efficiency remains constant, $\sim 98 \%$, up to few keV when the backward/forward anisotropy in the angular distribution starts to play an important role.

\subsection{Dead time and other corrections}

The dead time is the minimum time between two consecutive events that can be identified and separately reconstructed. Since the net effect of the dead time is a reduction in the number of recorded events, a correction has to be applied in order to compensate for the loss of counts. At $\mathrm{n}_{-} \mathrm{TOF}$, the use of flash ADCs for data acquisition, combined with a powerful pulse shape analysis procedure (see ref. [24]) are reducing the effect of the dead time to a few percent, provided that the sample thickness is properly chosen. The minimum time difference that allows identifying two consecutive signals is between $150 \mathrm{~ns}$ and $350 \mathrm{~ns}$, depending on the detection system. Nevertheless, considering the high count-rate and the need of reaching a high accuracy on the measured flux, a correction is necessary. To this end, consecutive events within a time window $\tau$ were discarded; then the resulting histogram was subsequently corrected for this fixed dead time $\tau$, assuming the non-paralyzable model [31] for neutron time-of-flight measurements. According to this model, when the counting losses are small, the number of corrected events, $C_{r}(T)$ as a function of time-of-flight $T$, is related to the measured one, $C_{m}(T)$, by means of

$$
C_{r}(T)=\frac{C_{m}(T)}{1-\sum_{t=T-\tau}^{T} \frac{C_{m}(t)}{n}},
$$

where $n$ is the number of neutron pulses or bunches and the sum runs of the preceding (fractional) bins covering a time $\tau$.

Due to the high instantaneous flux at EAR2, a correction on the dead time must be included in the case of SiMon2 and for both micromegas, although the sample mass were selected in order to minimise this effect. Only the PPACs are fast enough $(\tau<10 \mathrm{~ns})$ to have negligible dead-time corrections in the energy range covered with these detectors. Figure 7 shows the value of the dead-time correction for these detectors as a function of the neutron time-of-flight. At the thermal peak $(\approx 6 \mathrm{~ms})$, the correction is close to $1 \%$ while above $200 \mathrm{keV}(3 \mu \mathrm{s})$ the corrections increase up to $10 \%$. The uncertainty on the dead time correction is treated as an energy-dependent effect. It has been estimated by calculating the dead-time correction for artificially increased time separations between two pile-up signals.

Other corrections that have to be considered in the extraction of the flux are related to the attenuation of the 


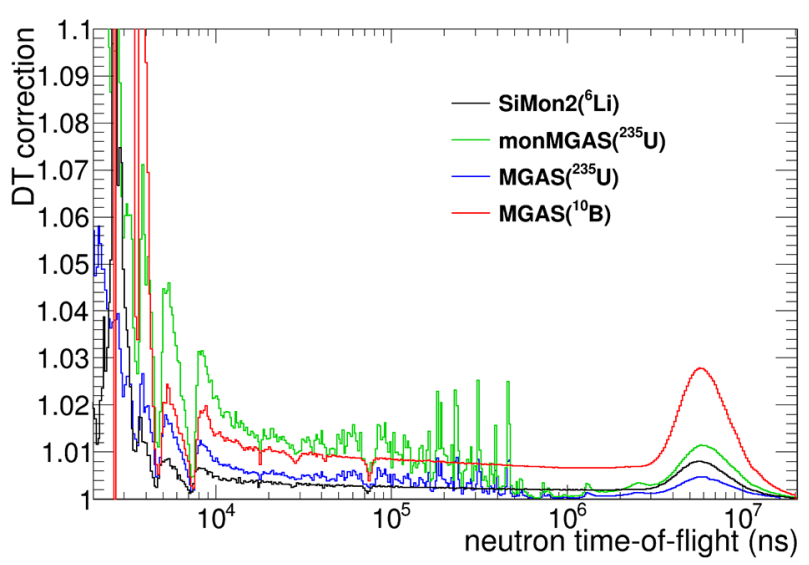

Fig. 7. Dead time correction factor for MGAS, monMGAS and SiMon2 detectors. The count rate of the detector is multiplied by this factor in order to get the real number of events produced in the sample.

neutron beam in the various windows, electrodes and deposits placed upstream of the sample under analysis, as well as the self-shielding effect in the respective deposit. The neutron beam attenuation, estimated by means of Monte Carlo simulations, is small, typically less than $2 \%$ at thermal energy if only the windows and electrodes are in the beam. However, it becomes higher, although never exceeding a few percent, when thick ${ }^{6} \mathrm{Li}$ or ${ }^{10} \mathrm{~B}$ deposits are in the beam, due to the high ( $n$, tot) cross sections of these isotopes. Self-shielding effects were corrected by means of the classical analytical formula, taking into account the tabulated total cross section. Apart from the energy independent factors, like the sample mass, the uncertainties on the transmission and self-absorption corrections are included in the uncertainty due to systematic effects associated with the Monte Carlo simulations, of at most $2 \%$, considering also that all cross sections involved are convoluted, through the yield, with the response function using the method described in sect. 3 .

\subsection{Analysis of the activation measurement}

An alternative to determine the absolute value of the neutron flux at a specific energy is the double foil activation method. It relies on the use of two identical foils made of the same material (isotope) which presents one large and isolated resonance; the foil thickness is selected in such a way that the first one depletes entirely the neutron fluence at the position of the resonance leaving the rest of the spectrum untouched [32].

In the present activation measurement, two gold foils were exposed at the same time to the neutron beam for $\simeq 1$ hour. After the activation the number of counts corresponding to the $411.8 \mathrm{keV} \gamma$ line were measured separately in the same geometry for the two foils using a $\mathrm{LaBr}_{3}$ scintillator detector. The number of ${ }^{198} \mathrm{Au}$ nuclei produced by activation was obtained and the neutron flux deduced from the difference in counts, taking into account the difference in counts for each sample, the geometric efficiency of the setup used and the time of exposure to the neutron beam.

The $100 \mu \mathrm{m}$ thick foils resulted in a flux-integrated activation of the first foil by the incident neutron flux, and activation of the second foil by the transmitted neutron flux. From the calculated expected flux-dependent difference in activation we observed that about $80 \%$ of the difference was due to the strong ${ }^{197} \mathrm{Au}(n, \gamma)$ resonance at $4.9 \mathrm{eV}$, and roughly $10 \%$ due to the lower energy neutrons down to subthermal energies, and $10 \%$ due to higher energy neutrons.

\section{Results}

The comparison and combination of all measurements described above allowed us to determine the neutron flux in EAR2, in particular its energy dependence, from $2 \mathrm{meV}$ to $100 \mathrm{MeV}$. The results of all measurements are shown in fig. 8. For convenience, the flux is expressed in units of lethargy and per nominal pulse of $7 \cdot 10^{12}$ protons. Since each measurement is affected by a few percent uncertainty on the absolute value of the flux, all measurements were re-normalized at thermal neutron energy, where the cross sections of all three reactions used in the measurements are well known, therefore considered standard. It was decided to take the SiMon2 measurement as the reference because the sample mass and the efficiency, which affect the absolute value of the flux, are best characterised in this case.

In addition, a comparison between the absolute value determined by the activation measurement and the results from the SiMon2 detector has been performed, showing an agreement within the quoted error bars of $5 \%$ (fig. 9).

The various experimental results shown in fig. 8 were combined, after re-normalization at thermal neutron energy, in order to extract an evaluated neutron flux. The weighted average was performed taking into account the statistical uncertainty as the weighting factor, while uncertainties due to systematic effects were treated separately (as discussed later on). The data to be included in the combination in different neutron energy regions were chosen mostly on the basis of the underlying cross section, i.e. whether it is considered standard in that region. In table 2, a summary of measurements included in the evaluation for several energy regions is presented. The agreement between the various measurements below $10 \mathrm{keV}$ is in general very good. Systematic effects of the energy dependence have been estimated from the comparison of the different measurements. As an example, the ratio between MGAS $\left({ }^{10} \mathrm{~B}\right)$ and SiMon2 shows a smooth behaviour within the statistical uncertainties (fig. 10), with the ratio never departing from the $2 \%$ mark in the whole energy region of the comparison.

Above $10 \mathrm{keV}$, in principle the flux extracted from the ${ }^{235} \mathrm{U}(n, f)$ reaction should not be considered in the analysis, as the cross section of this reaction is not considered standard from thermal up to $150 \mathrm{keV}$. Nevertheless, in order to increase the statistical accuracy in this region, those data have been included in the flux evaluation at 


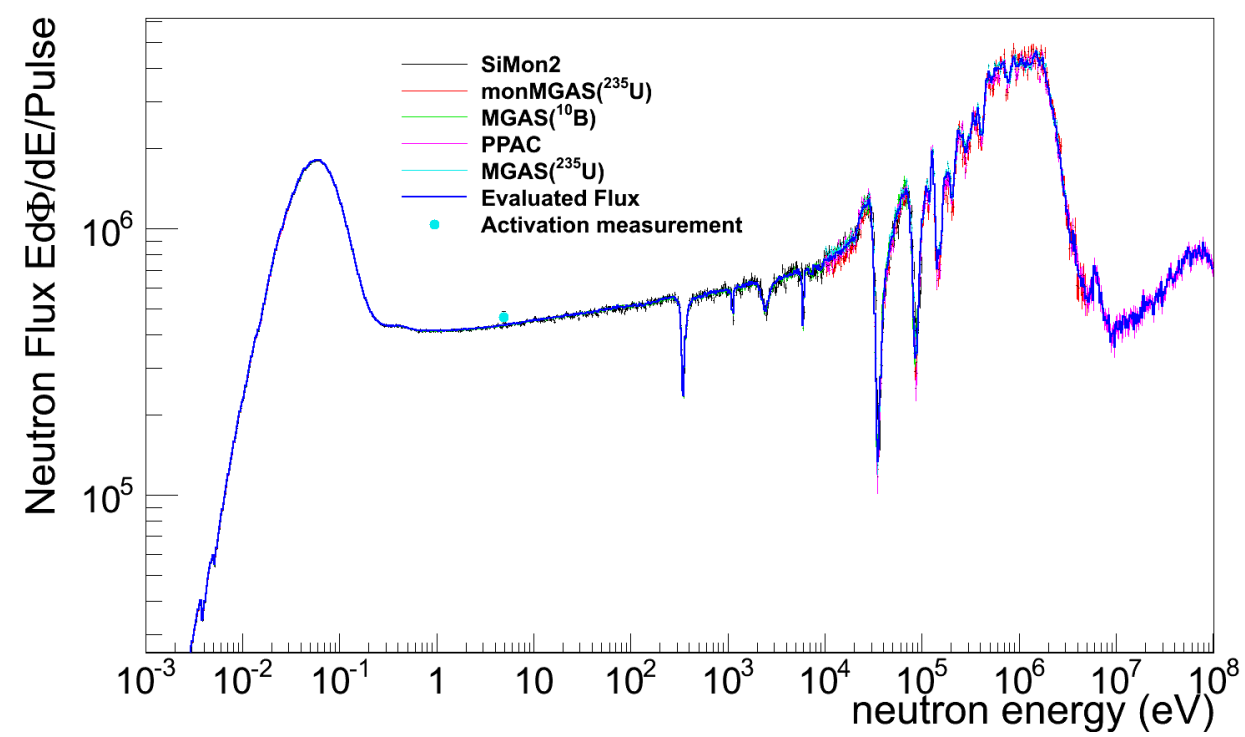

Fig. 8. Results from all measurements performed for the neutron flux determination and considered in the present analysis. The thick blue line corresponds to the evaluated flux. Each result has been normalized close to the thermal point to the value provided by SiMon2 measurement, while the cyan dot represents the integral measurement from the flux-averaged activation measurement.

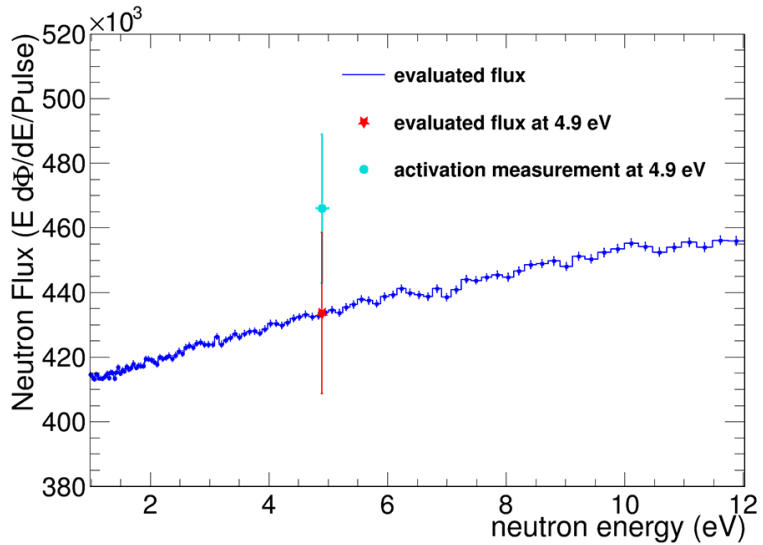

Fig. 9. Comparison of the activation measurement (cyan dot) with the value of the evaluated flux (red dot) at $4.9 \mathrm{eV}$. Results are in agreement within overall uncertainties of $5 \%$.

the expenses of a slightly higher uncertainty. In fact, as can be noted in fig. 8, the flux based on the ${ }^{235} \mathrm{U}(n, f)$ reaction in the $10-30 \mathrm{keV}$ neutron energy range is systematically lower than the one obtained from the other two reactions. This effect was already observed in ref. [12] and it is associated with an overestimation of the evaluation cross section in the ENDF/B-VII.1 library which is pending of corrections for the next coming ENDF/B-VIII release.

At $100 \mathrm{keV}$, the SiMon2 detector starts to be limited by the $\gamma$-flash. Therefore, above this energy the neutron flux is evaluated on the basis of the micromegas and PPAC results alone. A similar argument applies to the MGAS and monMGAS detectors, which start to be affected by the $\gamma$-flash around $5 \mathrm{MeV}$. Hence, the only detector that allows reaching $100 \mathrm{MeV}$ neutron energy is the PPAC (see fig. 11), thanks to the low sensitivity to the $\gamma$-flash and its very fast signals.

Figure 12 shows the dependence of the statistical uncertainties with the neutron energy. Up to $5 \mathrm{MeV}$ it turns to be less than $2 \%$ except at the position of the dips in the flux; while above this energy the statistical uncertainty increases up to $8.5 \%$ because only the PPAC data have been included.

An interesting feature is observed at very low energy. For the first time at $\mathrm{n}_{-} \mathrm{TOF}$ it was in fact possible to detect neutrons with an energy as low as $2 \mathrm{meV}$, thanks to the combination of a larger memory on the Flash ADC and the shorter time-of-flight, which provides the possibility to perform measurements at sub-thermal neutron energies. The flux at those energies shows the typical solid state effect of neutron diffraction, i.e., the Bragg scattering of neutrons in the source and the beam line materials. Figure 13 exhibits a structure in the evaluated flux related with this effect thanks to the good energy resolution of the neutron beam and detection systems. The Braggedge transmission dips in the flux, related to the scattering off a crystal plane, like polycrystalline metal, is visible at around 3.7 and $5.0 \mathrm{meV}$. They are due to the in-beam aluminum windows near the spallation target. The positions correspond to the measured transmission of $\mathrm{Al}$ [33].

\subsection{Uncertainties due to systematic effects}

The uncertainties on several energy-dependent corrections are uncorrelated for the detector values at a given energy, but correlated for a particular detector over the entire energy range. We refer to them as systematic uncertainties.

The systematic uncertainties in the shape of the flux are a combination of several energy-dependent contributions. The main ones are the uncertainty on the evaluated 
Table 2. Summary of the detector used for the n_TOF-EAR2 neutron flux evaluation in different energy ranges up to $100 \mathrm{MeV}$.

\begin{tabular}{|c|c|c|c|c|c|c|}
\hline Energy range & $25 \mathrm{meV}$ & $<10 \mathrm{keV}$ & $10 \mathrm{keV}-100 \mathrm{keV}$ & $100 \mathrm{keV}-1 \mathrm{MeV}$ & $1-5 \mathrm{MeV}$ & $100 \mathrm{MeV}$ \\
\hline SiMon2 $\left({ }^{6} \mathrm{Li}\right)$ & yes & yes & yes & - & - & - \\
\hline $\operatorname{monMGAS}\left({ }^{235} \mathrm{U}\right)$ & yes & - & yes & yes & yes & - \\
\hline $\operatorname{MGAS}\left({ }^{235} \mathrm{U}\right)$ & - & - & yes & yes & - & - \\
\hline $\operatorname{MGAS}\left({ }^{10} \mathrm{~B}\right)$ & yes & yes & yes & - & - & - \\
\hline $\operatorname{PPAC}\left({ }^{235} \mathrm{U}\right)$ & yes & - & yes & yes & yes & yes \\
\hline
\end{tabular}

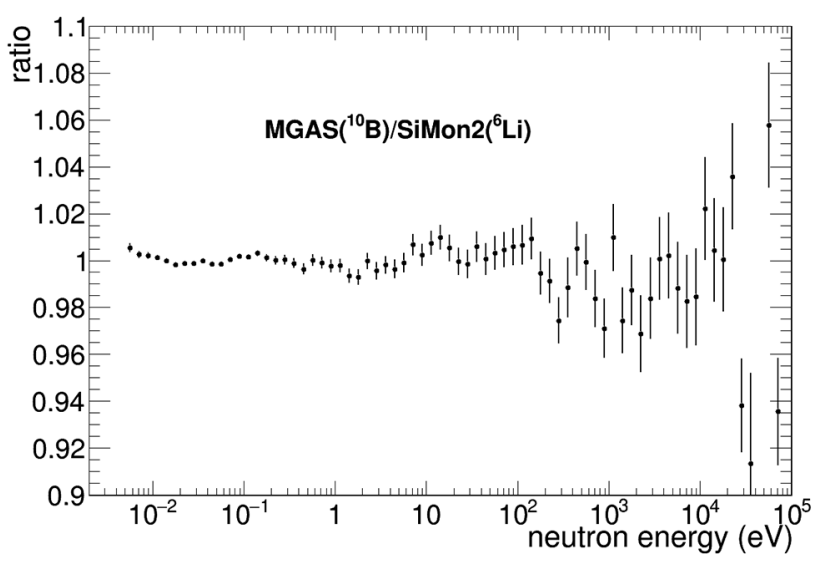

Fig. 10. Ratio of the ${ }^{10} \mathrm{~B}(n, \alpha)$ data taken with MGAS to the reference ones, taken with $\operatorname{SiMon} 2$, and based on the ${ }^{6} \operatorname{Li}(n, t)$ reaction. Data points are shown with their statistical uncertainties. Up to $20 \mathrm{keV}$, the dispersion is in the order of $2 \%$.

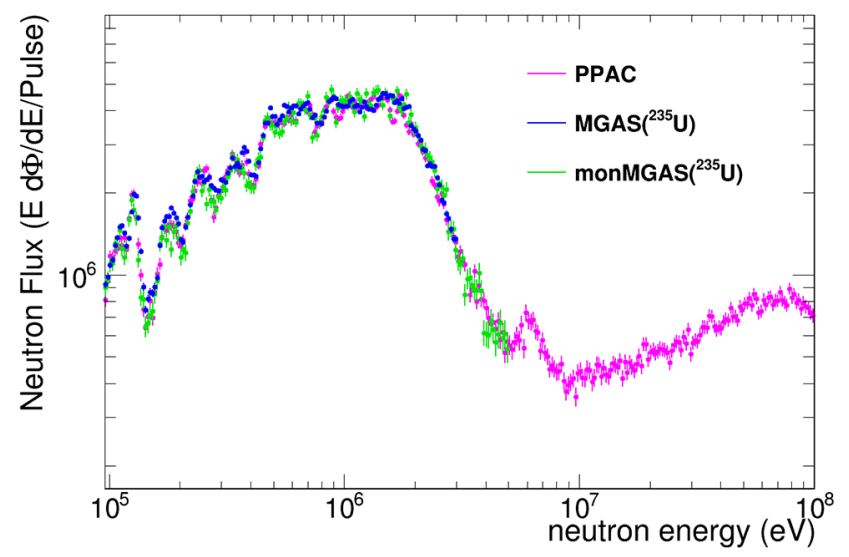

Fig. 11. Comparison of the experimental data above $100 \mathrm{keV}$ : PPAC (pink), MGAS $\left({ }^{235} \mathrm{U}\right)$ (blue), and monMGAS $\left({ }^{235} \mathrm{U}\right)$ (green). The difference between the various data in this energy region is less than $8 \%$.

cross section used to extract the flux, on the dead time correction and on the detector efficiency, which in turn depends on the thresholds and corrections applied in data analysis as well as on the angular distribution of the emitted products. Other sources of uncertainty are related to the corrections for the neutron beam attenuation in the windows and other materials in the beam, and to the neutron background inside the experimental area.

The various components have been estimated for each detector and energy region, and added in quadrature to

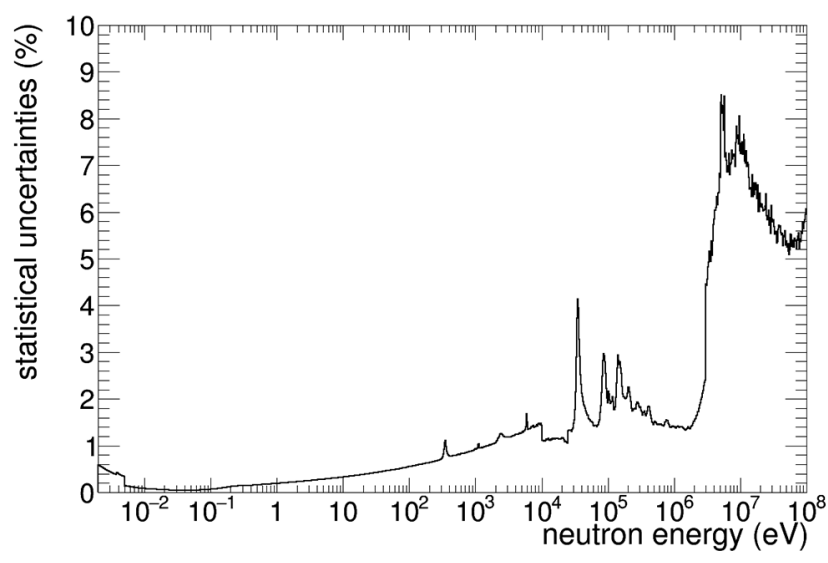

Fig. 12. Statistical uncertainties on the flux as a function of the neutron energy.

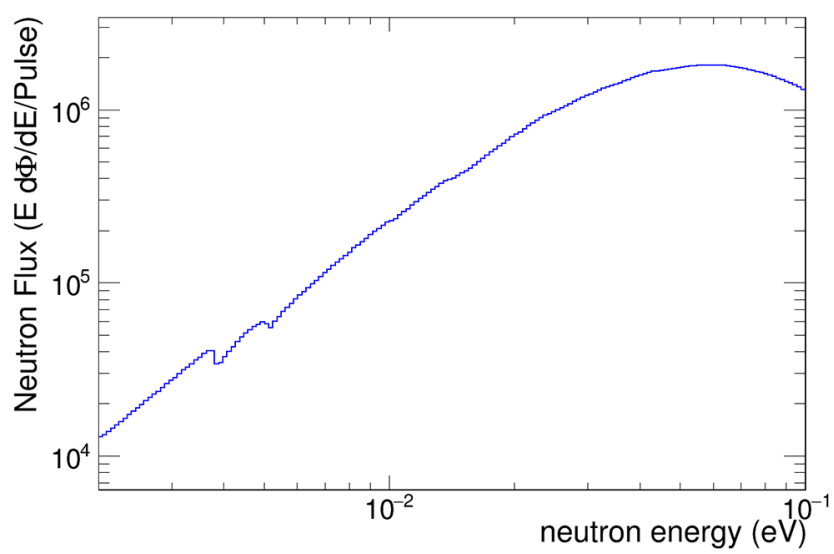

Fig. 13. Evaluated neutron flux in the sub-thermal region. The dips in the flux are caused by neutron Bragg scattering.

extract the overall systematic uncertainty for every data set. The estimated systematic uncertainties on each data set in different neutron energy regions are summarized in table 3 . When the evaluated flux is obtained by a combination of different results (i.e., from different detectors), the estimated systematic uncertainty affecting each dataset is calculated as $\sqrt{1 / \sum \sigma_{i}^{2}}$ in the combination, providing an a priori systematic uncertainty on the evaluated flux. The corresponding values are reported in the last line of table 3 .

The reliability of the obtained values has been subsequently checked by comparing each dataset with the evaluated neutron flux to verify that the differences were statis- 
Table 3. Summary of the uncertainties due to systematic effects for all datasets used in the EAR2 neutron flux evaluation, for different energy regions up to $100 \mathrm{MeV}$. Although the ${ }^{235} \mathrm{U}(n, f)$ cross section is considered standard at the thermal energy and in the range $0.15-200 \mathrm{MeV}$, data based on this reaction have been considered also in the $10-150 \mathrm{keV}$ region, in order to increase the statistical accuracy. The last line represents the overall estimated systematic uncertainty on the energy dependence of the evaluated flux, calculated as the average of the uncertainties of every set of data used in the evaluation: $\sqrt{\sum \sigma_{i}^{2}} / N$ with $N$ the number of datasets.

\begin{tabular}{|c|c|c|c|c|c|c|}
\hline Energy range & $25 \mathrm{meV}$ & $<10 \mathrm{keV}$ & $10-100 \mathrm{keV}$ & $100 \mathrm{keV}-1 \mathrm{MeV}$ & $1-5 \mathrm{MeV}$ & $>5 \mathrm{MeV}$ \\
\hline $\operatorname{SiMon} 2\left({ }^{6} \mathrm{Li}\right)$ & 2.4 & 2.4 & 9.3 & - & - & - \\
\hline $\operatorname{monMGAS}\left({ }^{235} \mathrm{U}\right)$ & 3.0 & - & 5.0 & 4.5 & 7.7 & - \\
\hline $\operatorname{MGAS}\left({ }^{235} \mathrm{U}\right)$ & - & - & 4.5 & 3.2 & - & - \\
\hline $\operatorname{MGAS}\left({ }^{10} \mathrm{~B}\right)$ & 3.0 & 3.1 & 3.0 & - & - & - \\
\hline $\operatorname{PPAC}\left({ }^{235} \mathrm{U}\right)$ & 3.0 & - & 6.8 & 5.5 & 5.5 & 6.2 \\
\hline Uncertainty on evaluated flux (\%) & 1.4 & 2.0 & 2.7 & 2.6 & 4.7 & 6.2 \\
\hline
\end{tabular}

tically compatible within the total uncertainties. A further check has been performed by calculating the dispersion of the used datasets relative to the weighted average, and comparing it to the error on the weighted average calculated considering only the statistical errors. In all cases, the deviations are compatible with the estimated systematic uncertainties. In some energy regions, however, only one dataset is used in the neutron flux evaluation, so that one must rely on the a priori estimate.

The uncertainties reported in table 3 refer only to the shape of the flux. Typically, it is not mandatory to also know with high accuracy the absolute value of the flux, since in each measurement an independent normalization is achieved (for example through the saturated resonance technique in capture cross section measurements, or with reference to ${ }^{235} \mathrm{U}$ or ${ }^{238} \mathrm{U}$ in fission reactions). Nevertheless, it may be useful to extract from the data the absolute value, for example for a meaningful comparison with Monte Carlo simulations of the spallation source.

The systematic uncertainty on the absolute value of the flux is mainly related with the uncertainty on the mass of the converter deposit, the absolute value of the efficiency, rather than its energy dependence, and the determination of the proton beam intensity. As mentioned earlier, only the SiMon2 results were considered for absolute normalization, as this setup was less affected by uncertainties in the deposit mass and efficiency, estimated to be overall of the order of $5 \%$.

\subsection{Simulations}

The evaluated EAR2 neutron flux has been compared with the results of Monte Carlo simulations of the $\mathrm{n}_{-} \mathrm{TOF}$ spallation neutron source obtained with FLUKA [26] and GEANT4 [27]. The geometry implemented in the simulations and the physics list used in GEANT4 are described in detail in $[8,28]$. In both cases, neutrons produced in the spallation target and exiting from the top are resampled and propagated through the various elements along the beam line, in particular the two collimators, up to the experimental area. The neutron energy is extracted from the

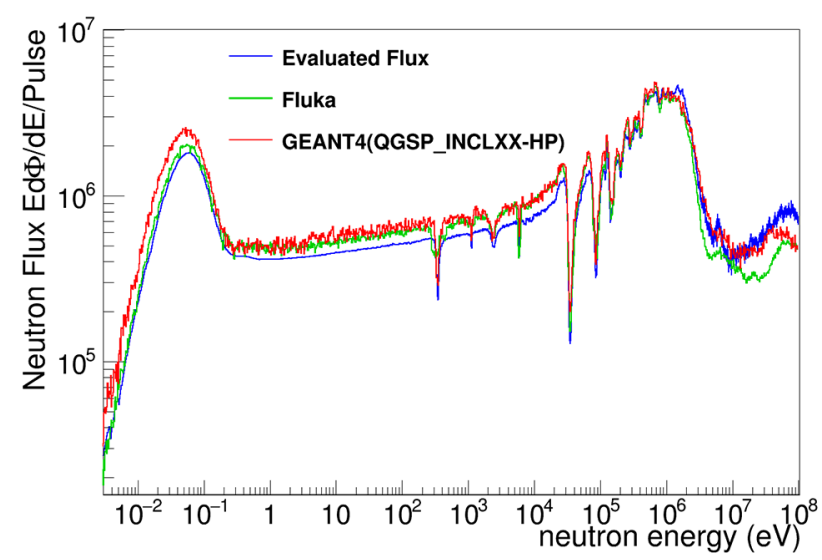

Fig. 14. The EAR2 evaluated neutron flux (blue) in the whole energy range compared with the results of FLUKA (green) and GEANT4 (red) simulations of the spallation process, and subsequent resampling and propagation of emitted neutrons to the experimental area.

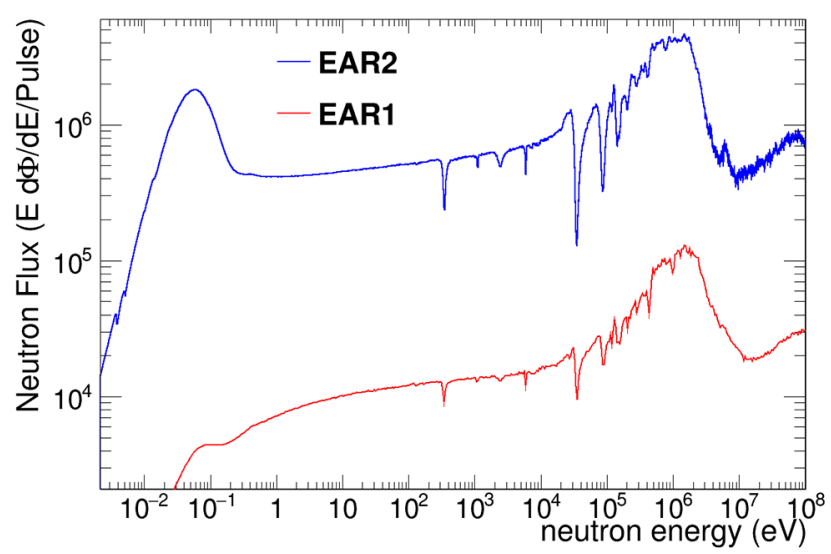

Fig. 15. Comparison between the evaluated neutron flux in EAR2 (blue) and in EAR1 (red). The increase at the new measuring station is on average a factor 40 .

total neutron time-of-flight, i.e. from the start of the spallation process to the arrival of the neutron at the sample position in EAR2. 
The comparison between the measured neutron flux and the simulations is shown in fig. 14. As already observed for the flux in EAR1 [34], the shape is reproduced reasonably well by both codes, but in general simulations tend to overestimate the absolute value of the neutron flux, by approximately $20 \%$. In particular, while the two simulations show a very similar trend between $100 \mathrm{meV}$ and $1 \mathrm{MeV}$, FLUKA reproduces more closely the thermal peak, while GEANT4 shows a better agreement with the evaluated flux at higher energy, i.e. above $1 \mathrm{MeV}$. The origin of the inconsistencies among the simulations and with the experimental result are unclear, and require more detailed investigations on the Monte Carlo side. One possible explanation is related to the effect of small details in material and position of the different elements in the beam line, although it has recently been suggested that the systematic overestimate in the neutron flux could be related to pion production in the spallation reactions [35].

\section{Conclusions}

After the completion of the second experimental area at n_TOF, a commissioning program was carried out by the Collaboration with the aim of determining with good accuracy the characteristics of the neutron beam, i.e. flux, spatial beam profile, resolution function, et cetera. The results of the commissioning in terms of neutron flux, in particular its shape as a function of neutron energy, have been reported in this paper. The combination of three different detector systems, SiMon2, micromegas and PPAC, based on different operational concepts, and exploiting three different neutron-converting reactions with standard cross sections, i.e. ${ }^{10} \mathrm{~B}(n, \alpha),{ }^{6} \mathrm{Li}(n, t)$ and ${ }^{235} \mathrm{U}(n, f)$, has provided the energy dependence of the neutron flux in EAR2 with an uncertainty due to systematic effects between 2 and $6 \%$, in an energy range which extends from $2 \mathrm{meV}$ to $100 \mathrm{MeV}$ [36]. The absolute value was determined on the basis of the most accurate result, obtained with the SiMon2 detector, and was used as reference for all other measurements. This result was compared with the flux determined via activation of a ${ }^{197} \mathrm{Au}$ sample. The observed agreement within few percents confirms the quoted uncertainty on the absolute value of the flux of around 5\%. In addition, a comparison between the evaluated neutron flux in EAR2 and in EAR1 shows on average a 40 times higher neutron flux at the new measuring station, as shown in fig. 15 .

The evaluated flux in EAR2 has been also compared with the results of Monte Carlo simulations, performed with FLUKA and GEANT4 of the n_TOF neutron spallation source, showing a reasonable agreement in the whole energy range. Some minor differences are observed among the two simulations and with the measured flux, possibly indicating some minor shortcomings in the simulation physics/geometry or in the neutron propagation procedure.

The evaluated neutron flux reported here is an important quantity for the planning and execution of cross sec- tion measurements in EAR2 at $\mathrm{n}_{-} \mathrm{TOF}$ and a fundamental prerequisite for collecting high quality data. Apart from the measurements of this work, the neutron flux is continuously monitored during the various measurements, with the SiMon2 and micromegas detectors, and is verified with a dedicated measurement at the beginning of each experimental campaign.

The authors acknowledge support from the European Atomic Energy Communitys (Euratom) Seventh Framework Program FP7/2007-2011 under the Project CHANDA (Grant No. 605203). We acknowledge the support by the Narodowe Centrum Nauki (NCN), under the grant UMO2012/04/M/ST2/00700 and UMO-2016/22/M/ST2/00183. We acknowledge the support by the Croatian Science Foundation under Project No. 1680.

\section{References}

1. N. Colonna et al., Energy Environ. Sci. 3, 1910 (2010).

2. OECD/NEA WPEC Subgroup 26 Final Report, Uncertainty and Target Accuracy Assessment for Innovative Systems Using Recent Covariance Data Evaluations, NEA No. 6410 (2008), http://www.nea.fr/html/science/wpec/ volume26.

3. G. Wallerstein et al., Rev. Mod. Phys. 69, 995 (1997).

4. F. Käppeler, R. Gallino, S. Bisterzo, Wako Aoki, Rev. Mod. Phys. 83, 157 (2011).

5. I. Porras et al., Nucl. Data Sheets 120, 246 (2014).

6. M. Sabaté-Gilarte et al., Rep. Pract. Oncol. Radiother. 21, 113 (2016).

7. C. Guerrero et al., Eur. Phys. J. A 49, 27 (2013).

8. C. Weiss et al., Nucl. Instrum. Methods A 799, 90 (2015).

9. S. Barros et al., J. Instrum. 10, 09003 (2015).

10. M. Barbagallo et al., Phys. Rev. Lett. 117, 152701 (2016).

11. ICRU, International Commission on Radiation Units and Measurements, report 60, issue 30, December 1998.

12. M. Barbagallo et al., Eur. Phys. J. A 49, 156 (2013).

13. A.D. Carlson et al., Nucl. Data Sheets 110, 3215 (2009).

14. A.D. Carlson, Metrologia 48, S328 (2011).

15. L. Cosentino et al., Rev. Sci. Instrum. 86, 073509 (2015).

16. S. Marrone et al., Nucl. Instrum. Methods A 517, 389 (2004).

17. I. Giomataris et al., Nucl. Instrum. Methods A 376, 29 (1996).

18. S. Andriamonje et al., J. Korean Phys. Soc. 59, 1597 (2011).

19. I. Giomataris, R. De Oliveira, Patent CEA-CERN, Application Number 09290825.0 (2009), Method for fabricating an amplification gap of an avalanche particle detector.

20. C. Paradela et al., Phys. Rev. C 82, 034601 (2010).

21. D. Tarrío et al., Phys. Rev. C 83, 044620 (2011).

22. D. Tarrío, Neutron-induced fission fragment angular distribution at CERN n_TOF: The Th-232 case, $\mathrm{PhD}$ Thesis (2012).

23. Y.H. Chen, L. Tassan-Got, L. Audouin et al., EPJ Web of Conferences 146, 03020 (2017).

24. P. Žugec et al., Nucl. Instrum. Methods A 812, 134 (2016).

25. M.B. Chadwick et al., Nucl. Data Sheets 112, 2887 (2011).

26. A. Fasso et al., Technical Report CERN-2005-10, INFN/TC_05/11, SLAC-R-73 (2005), Fluka: A multiparticle transport code. 
27. J. Allison et al., Nucl. Instrum. Methods A 835, 186 (2016).

28. J. Lerendegui et al., Eur. Phys. J. A 52, 100 (2016).

29. F. Gunsing et al., Phys. Rev. C 85, 064601 (2012).

30. F.J. Hambsch, I. Ruskov, Nucl. Sci. Eng. 163, 1 (2009).

31. M.S. Moore, Nucl. Instrum. Methods 169, 245 (1980).

32. C. Borcea et al., Nucl. Instrum. Methods Phys. Res. A 513, 524 (2003)
33. R.J. Brown, L.M. Bollinger, Nucl. Sci. Eng. 4, 576 (1958) https://www.oecd-nea.org/janisweb/exfor/11468. 002.

34. S. Lo Meo et al., Eur. Phys. J. A 51, 160 (2015).

35. D. Mancusi et al., Eur. Phys. J. A 53, 80 (2017).

36. https://twiki.cern.ch/twiki/bin/view/NTOFPublic/ NTOF PublicEAR2Flux. 


\section{Production Queries for the Author}

AUTHOR: Please check VERY CAREFULLY throughout

1) TEXT (Copy-editing corrections already inserted in the manuscript-to-proofs process)

2) FIGURES (if any): All writings and symbols. Thank you 